\title{
Treatment of patients with gastro-entero-pancreatic (GEP) tumours with the novel radiolabelled somatostatin analogue [177 Lu-DOTA ${ }^{0}$, Tyr $^{3}$ ]octreotate
}

\author{
D.J. Kwekkeboom¹, W.H. Bakker ${ }^{1}$, B.L. Kam¹, J.J.M. Teunissen1, P.P.M. Kooij ${ }^{1}$, W.W. Herder², R.A. Feelders², \\ C.H.J. Eijck ${ }^{3}$, M. Jong ${ }^{1}$, A. Srinivasan ${ }^{4}$, J.L. Erion ${ }^{4}$, E.P. Krenning ${ }^{2}$ \\ 1 Department of Nuclear Medicine, Erasmus Medical Center, Rotterdam, The Netherlands \\ 2 Department of Internal Medicine, Erasmus Medical Center, Rotterdam, The Netherlands \\ 3 Department of Surgery, Erasmus Medical Center, Rotterdam, The Netherlands \\ 4 Mallinckrodt Medical, St. Louis, MissouriUSA
}

Received: 17 July 2002 / Revised: 12 October 2002 / Published online: 9 January 2003

(C) Springer-Verlag 2003

\begin{abstract}
Medical treatment and chemotherapy are seldom successful in achieving objective tumour reduction in patients with metastatic neuroendocrine tumours. Treatment with the radiolabelled somatostatin analogue $\left[{ }^{90} \mathrm{Y}^{-D O T A}{ }^{0}, \mathrm{Tyr}^{3}\right]$ octreotide may result in partial remissions in $10-25 \%$ of patients. The newer analogue $\left[\mathrm{DOTA}^{0}, \mathrm{Tyr}^{3}\right.$ ] ${ }^{2}$ ctreotate (octreotate) has a ninefold higher affinity for the somatostatin receptor subtype 2 as compared with $\left[\mathrm{DOTA}^{0}, \mathrm{Tyr}^{3}{ }^{3}\right.$ octreotide. Also, labelled with the beta- and gamma-emitting radionuclide ${ }^{177} \mathrm{Lu}$, it has proved very successful in achieving tumour regression in animal models. The effects of ${ }^{177} \mathrm{Lu}$-octreotate therapy were studied in 35 patients with neuroendocrine gastro-entero-pancreatic (GEP) tumours who underwent follow-up for 3-6 months after receiving their final dose. Patients were treated with doses of 100,150 or $200 \mathrm{mCi}$ ${ }^{177} \mathrm{Lu}$-octreotate, to a final cumulative dose of 600 $800 \mathrm{mCi}$, with treatment intervals of 6-9 weeks. Nausea and vomiting within the first $24 \mathrm{~h}$ after administration were present in $30 \%$ and $14 \%$ of the administrations, respectively. WHO toxicity grade 3 anaemia, leucocytopenia and thrombocytopenia occurred after $0 \%, 1 \%$ and $1 \%$ of the administrations, respectively. Serum creatinine and creatinine clearance did not change significantly. The effects of the therapy on tumour size were evaluable in 34 patients. Three months after the final administration, complete remission was found in one patient $(3 \%)$, partial remission in $12(35 \%)$, stable disease in $14(41 \%)$ and progressive disease in seven $(21 \%)$, including three patients who died during the treatment period. Tumour
\end{abstract}

D.J. Kwekkeboom (区)

Department of Nuclear Medicine,

Erasmus Medical Center, Dr Molewaterplein 40,

3015 GD Rotterdam, The Netherlands

e-mail: kwekkeboom@nuge.azr.nl

Tel.: +31-10-4635963, Fax: +31-10-4635997 response was positively correlated with a high uptake on the octreoscan, limited hepatic tumour mass and a high Karnofsky Performance Score. Because of the limited efficacy of alternative therapies, many physicians currently adopt an expectant attitude when dealing with patients with metastatic GEP tumours. However, in view of the high success rate of therapy with ${ }^{177} \mathrm{Lu}$-octreotate and the absence of serious side-effects, we advocate its use in patients with GEP tumours without waiting for tumour progression.

Keywords: Somatostatin receptor - Octreotate - Radionuclide therapy - Lutetium-177 - GEP tumours

Eur J Nucl Med (2003) 30:417-422

DOI 10.1007/s00259-002-1050-8

\section{Introduction}

Neuroendocrine gastro-entero-pancreatic (GEP) tumours, which comprise pancreatic islet cell tumours, non-functioning neuroendocrine pancreatic tumours and carcinoids, are usually slow growing. If the tumour is localized, the therapy of choice is surgery. When a metastatic tumour causes a syndrome by hormonal overproduction (i.e. carcinoid syndrome, hypergastrinaemia), treatment with somatostatin analogues results in symptomatic relief in most cases. In terms of objective tumour reduction (complete and partial remission), however, treatment with somatostatin analogues is seldom successful, whether or not it is given in combination with interferon- $\alpha[1,2,3]$.

A new development in cytoreductive therapy for GEP tumours is the use of radiolabelled somatostatin analogues. Initial studies with high doses of $\left[{ }^{111} \mathrm{In}-\right.$ 
DTPA $\left.^{0}\right]_{\text {octreotide ( }}{ }^{111}$ In-octreotide; Octreoscan) in patients with metastatic neuroendocrine tumours were encouraging, although partial remissions were exceptional $[4,5]$. However, ${ }^{111}$ In-coupled peptides are not ideal for peptide receptor radionuclide radiotherapy (PRRT) because of the small particle range and therefore short tissue penetration. Therefore, another radiolabelled so-

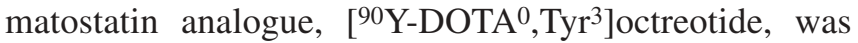
developed. Using this compound, partial remissions have been reported in $10-25 \%$ of patients with neuroendocrine tumours $[6,7,8]$.

Recently, it was reported that the somatostatin analogue $\left[\mathrm{DOTA}^{0}, \mathrm{Tyr}^{3}\right.$ ]octreotate has a ninefold higher affinity for the somatostatin receptor subtype 2 as compared with $\left[\mathrm{DOTA}^{0}, \mathrm{Tyr}^{3}\right]$ octreotide [9]. Also, labelled with the beta- and gamma-emitting radionuclide ${ }^{177} \mathrm{Lu}$, this compound was shown to be very successful in achieving tumour regression and animal survival in a rat model [10]. In a comparison in patients, we found that the uptake of radioactivity, expressed as a percentage of the injected dose of $\left.{ }^{177} \mathrm{Lu}^{-D O T A}{ }^{0}, \mathrm{Tyr}^{3}\right]$ octreotate $\left({ }^{177} \mathrm{Lu}\right.$-octreotate), was comparable to that after ${ }^{111}$ In-octreotide for kidneys, spleen and liver, but was three- to fourfold higher for four of five tumours [11]. We concluded that ${ }^{177} \mathrm{Lu}$-octreotate potentially represents an important improvement because of (a) the higher absorbed doses that can be delivered to most tumours with about equal doses to potentially dose-limiting organs and (b) the lower tissue penetration range of ${ }^{177} \mathrm{Lu}$ as compared with ${ }^{90} \mathrm{Y}$, which may be especially important for small tumours.

In this study we present the first data on the sideeffects as well as the antitumoral effects of ${ }^{177} \mathrm{Lu}-$ octreotate therapy in 35 patients with GEP tumours, who had a follow-up of 3-6 months after receiving their final dose.

\section{Materials and methods}

Patients. Thirty-five patients with GEP tumours were studied. All patients had tumour uptake on the octreoscan preceding the therapy that was at least as high as the uptake in the normal liver tissue. None of the patients had received prior treatment with other radiolabelled somatostatin analogues. Eight patients used Sandostatin s.c.; this medication was discontinued from 1 day before to 1 day after the treatment. Prerequisites for treatment were: $\mathrm{Hb} \geq 6 \mathrm{mmol} / \mathrm{l}, \mathrm{WBC} \geq 2 \times 10^{9} / 1$, platelets $\geq 80 \times 10^{9} / 1$, creatinine $\leq 150 \mu \mathrm{mol} / \mathrm{l}$ and Karnofsky Performance Score $\geq 50$.

All patients gave written informed consent to participate in the study, which was approved by the medical ethical committee of the hospital.

Methods. $\left[\mathrm{DOTA}^{0}, \mathrm{Tyr}^{3}\right.$ ]octreotate was obtained from Mallinckrodt, St Louis, Mo., USA. ${ }^{177} \mathrm{LuCl}_{3}$ was obtained from NRG, Petten, the Netherlands and Missouri University Research Reactor (MURR), Missouri, Mo., USA, and was distributed by IDB, Baarle-Nassau, the Netherlands. ${ }^{177} \mathrm{Lu}$-octreotate was prepared as described previously [11].
Granisetron $3 \mathrm{mg}$ was injected i.v. and an infusion of amino acids (lysine $2.5 \%$, arginine $2.5 \%$ in $110.9 \% \mathrm{NaCl} ; 250 \mathrm{ml} / \mathrm{h}$ ) was started $30 \mathrm{~min}$ before the administration of the radiopharmaceutical and lasted up to $3.5 \mathrm{~h}$ afterwards. The radiopharmaceutical was co-administered via a second pump system. The treatment doses of $100 \mathrm{mCi}$ were injected in $20 \mathrm{~min}$ and those of 150 and $200 \mathrm{mCi}$ were injected in $30 \mathrm{~min}$. The interval between treatments was 6-9 weeks. Patients were treated up to a cumulative dose of 750-800 mCi (27.8-29.6 GBq) (corresponding to a radiation dose to the bone marrow of $2 \mathrm{~Gy}$ ) [11], unless dosimetric calculations indicated that the radiation dose to the kidneys would then exceed $23 \mathrm{~Gy}$; in these cases the cumulative dose was reduced to 600-700 mCi.

Routine haematology, liver and kidney function tests, hormone measurements and serum tumour markers were measured 1 week prior to each therapy, as well as at follow-up visits. EORTC quality of life forms (QLQ-C30) [12] were filled out by the patients at each visit.

CT or MRI scanning was done within 3 months before the first therapy, and 6-8 weeks, 3 months and 6 months after the last treatment.

In vivo measurements. The tumours on the $\mathrm{CT}$ or MRI scans were measured and scored according to the WHO solid tumour response criteria. The uptake on the pretreatment octreoscans was scored visually on planar images on a 4-point scale: grade 1 , lower than normal liver tissue uptake; grade 2, equal to normal liver tissue uptake; grade 3, greater than normal liver tissue uptake; grade 4 , higher than normal spleen/kidney uptake.

Statistics. Analysis of variance (ANOVA), paired $t$ tests and chisquare tests were used. $P$ values $<0.05$ were considered significant.

\section{Results}

The study population comprised 14 men and 21 women with a mean age of 54 years(range 19-78 years). Twelve had carcinoid tumour, 12 neuroendocrine pancreatic tumour, 8 neuroendocrine tumour of unknown origin and 3 gastrinoma. Twelve patients had been operated in the past, 1 had had radiotherapy, 3 had had chemotherapy and 14 had been treated with octreotide (Sandostatin). Sixteen of the $35(46 \%)$ patients had documented progressive disease within 1 year before the start of the therapy. Cycle doses were $100 \mathrm{mCi}$ in seven patients, $150 \mathrm{mCi}$ in 14 and $200 \mathrm{mCi}$ in the remaining 14. Higher dosages were not administered, since $200 \mathrm{mCi}{ }^{177} \mathrm{LuCl}_{3}$ is typically bound to $180-300 \mu \mathrm{g}$ [DOTA $\left.{ }^{0}, \mathrm{Tyr}^{3}\right]$ octreotate, and higher doses would result in a lower percentage tumour uptake owing to receptor saturation. In $30 \mathrm{pa}-$ tients, the final intended cumulative dose of 600 $800 \mathrm{mCi}$ was administered. Three of the five remaining patients had progressive disease and died before completing their treatment; the other two patients, who were both elderly, stopped their treatment after reaching a cumulative dose of $600 \mathrm{mCi}$ because of the burden they felt the treatment to be.

Nausea and vomiting within the first $24 \mathrm{~h}$ after the administration were present in $30 \%$ and $14 \%$ of the admin- 

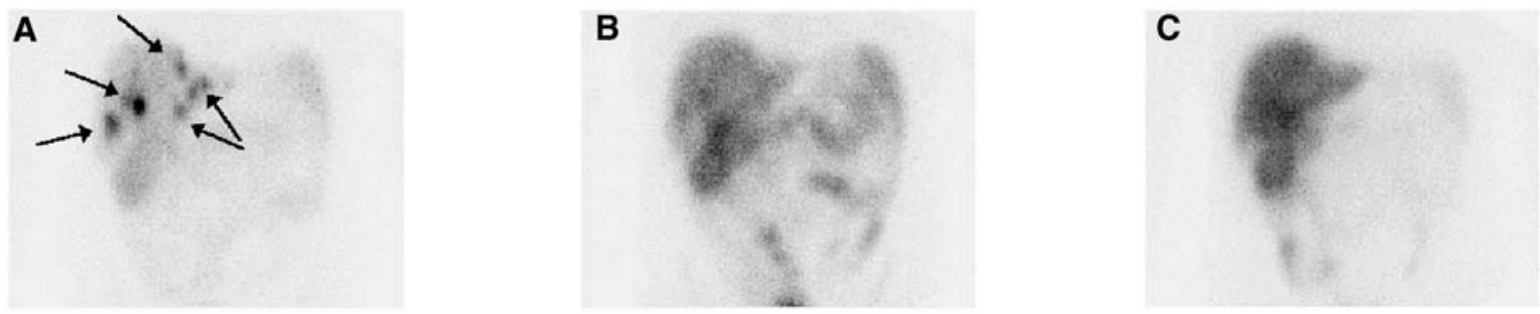

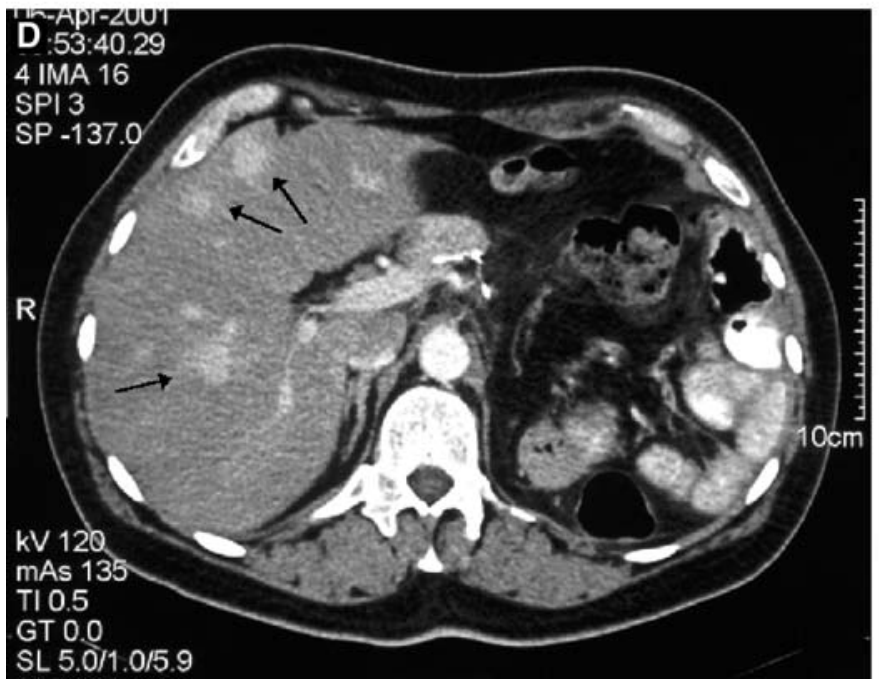

Fig. 1. A-C Planar scans of the abdomen, 3 days after the injection of $200 \mathrm{mCi}{ }^{177} \mathrm{Lu}$-octreotate in a patient with liver metastases of an operated neuroendocrine pancreatic tumour. A After the first treatment; $\mathbf{B}$ after the second treatment; $\mathbf{C}$ after the fourth treatment. Note the loss of intensity of uptake in the liver lesions (ar-

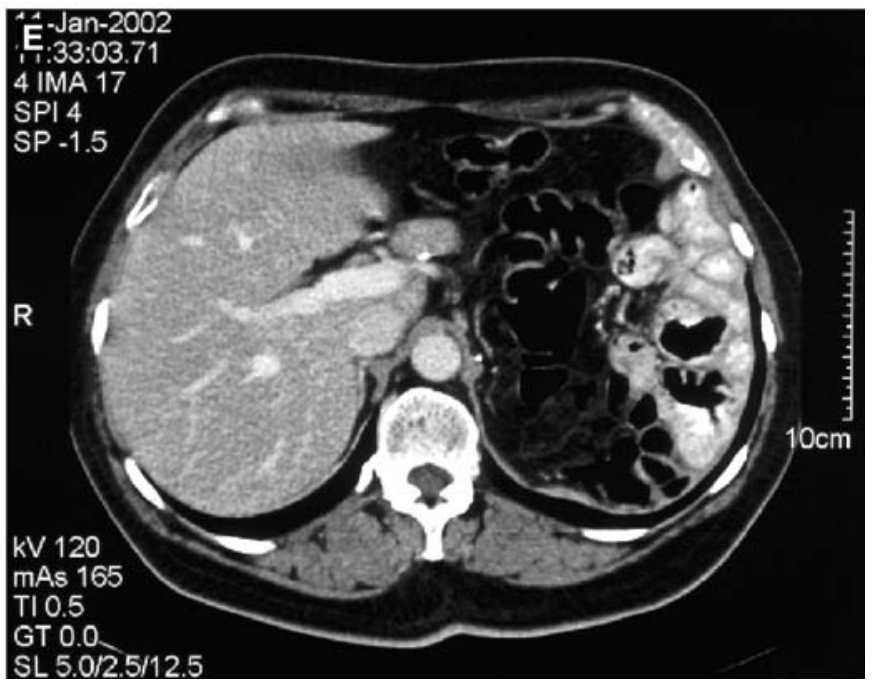

rows in A). This sign virtually always indicates a tumour volume response. D, E CT scans of the same patient: before treatment (D) and 3 months after the last treatment (E). Tumour (arrows in $\mathbf{D}$ ) is not demonstrated on E. Neither MRI nor octreoscan could demonstrate definite tumour deposits at that time
Table 1. Tumour responses in 34 patients, 3 months after the final administration of ${ }^{177} \mathrm{Lu}-$ octreotate. Three patients with PD died before reaching their final dose

\begin{tabular}{|c|c|c|c|c|c|}
\hline \multirow[t]{2}{*}{ Tumour type } & \multicolumn{4}{|c|}{ Response } & \multirow[t]{2}{*}{ Total } \\
\hline & $\mathrm{CR}$ & PR & SD & $\mathrm{PD}$ & \\
\hline Carcinoid & & $4(33 \%)$ & $6(50 \%)$ & $2(17 \%)$ & 12 \\
\hline NE pancreas & $1(8 \%)$ & $1(8 \%)$ & $7(58 \%)$ & $3(25 \%)$ & 12 \\
\hline NE unknown origin & & $4(57 \%)$ & $1(14 \%)$ & $2(29 \%)$ & 7 \\
\hline Gastrinoma & & $3(100 \%)$ & & & 3 \\
\hline Total & $1(3 \%)$ & $12(35 \%)$ & $14(41 \%)$ & $7(21 \%)$ & 34 \\
\hline
\end{tabular}

istrations, respectively, and were independent of the administered dose. Some abdominal pain was noticed during $11 \%$ of the administrations, especially in patients with liver metastases. Increased, mild hair loss was present in 17 of the 35 patients; hair regrowth had occurred at follow-up 3 and 6 months after the final administration.

WHO toxicity grade 2 or 3 anaemia ( $\mathrm{Hb} 4.95-6.2$ or 4.0-4.9 mmol/1, respectively), leucocytopenia (WBC $2.0-2.9$ or $1.0-1.9 \times 10^{9} / 1$, respectively) and thrombocytopenia (platelets $50-74.9$ or $25.0-49.9 \times 109 / 1$, respectively) occurred after $8 \%$ and $0 \%, 5 \%$ and $1 \%$, and $3 \%$ and $1 \%$ of the administrations, respectively. Toxicity grade 2 or 3 leucocytopenia or thrombocytopenia occurred in two out of three $(67 \%)$ patients who had had previous chemotherapy, as against seven out of 32 (22\%) patients who had not. Mean haematological parameters rose again during the follow-up after the final administration. Serum creatinine, creatinine clearance and serum $\mathrm{HbA} 1 \mathrm{c}$ did not change significantly (data not shown). In patients without thyroid hormone medication, serum TSH and $\mathrm{fT}_{4}$ levels did not change. In women, serum LH and FSH concentrations did not change significantly; in men, serum testosterone decreased and 
Fig. 2. A, B MRI scans in a woman with hepatic metastases of a gastrinoma. Before treatment (A) she was inoperable because of the size and localization of the metastases. Three months after completion of her treatment with $800 \mathrm{mCi}{ }^{177} \mathrm{Lu}-$ octreotate she had a PR (B). Note also that the hypertrophy of the gastric wall (arrow in $\mathbf{A}$ ) had diminished. Concomitantly, serum concentrations of gastrin (closed dots) and chromogranin-A (open dots) had decreased markedly $(\mathbf{C})$. At 6 months the MRI showed no further regression. The patient underwent left partial hepatectomy and the two right-sided lesions were drained and injected with alcohol. MRI 3 months after surgery showed further regression of the right-sided lesions
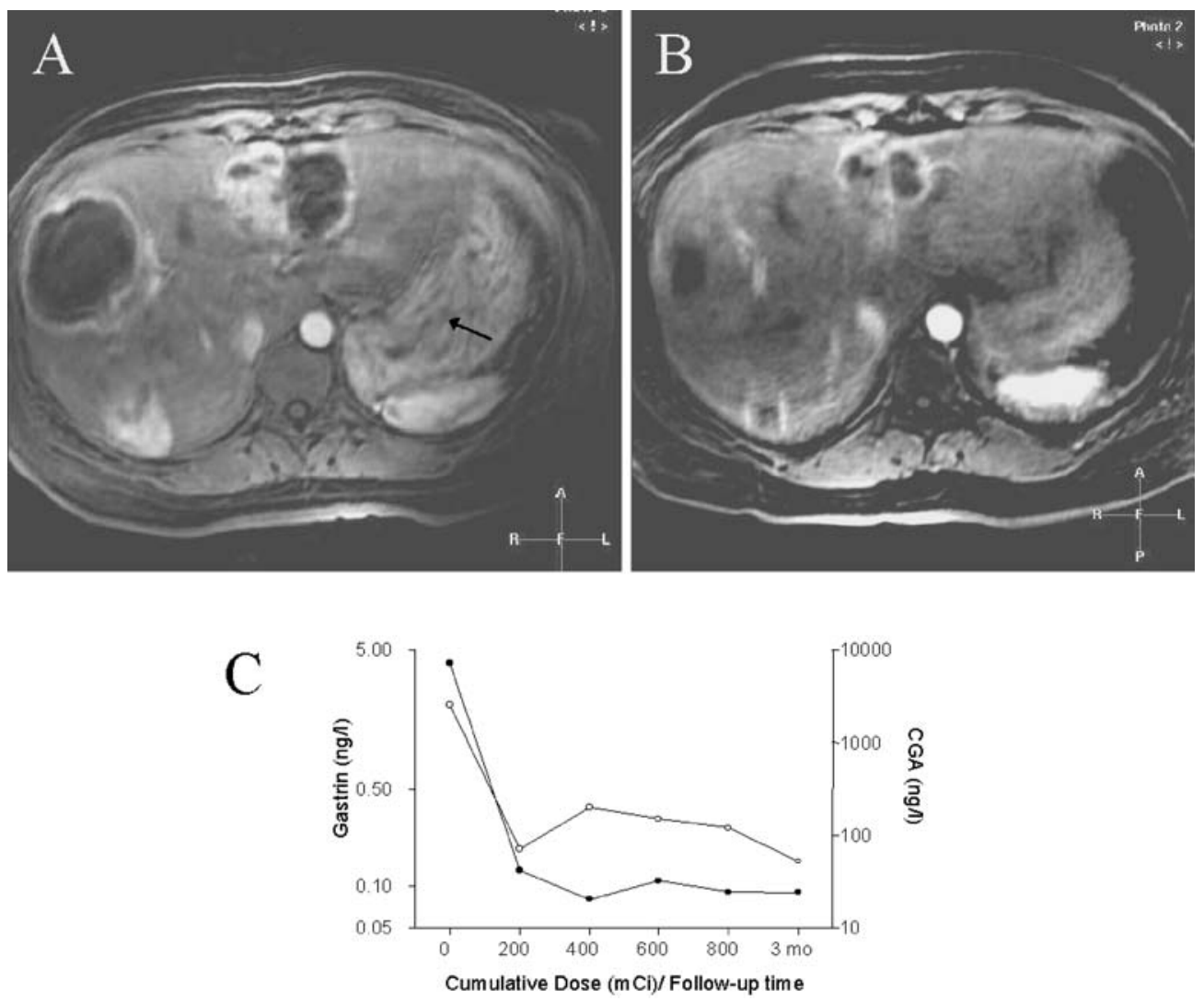

serum LH concentrations increased significantly. Also inhibin-B concentrations decreased and serum FSH levels increased significantly.

The effects of the therapy on tumour size were evaluable in 34 patients. Three months after the final administration (on average 9 months from the start of the treatment), a complete remission (CR), evaluated with CT scanning, MRI and somatostatin receptor imaging, was found in one patient (3\%), partial remission (PR) in 12 $(35 \%)$, stable disease (SD) in $14(41 \%)$ and progressive disease (PD) in 7 (21\%), including the three patients who died during the treatment period (Table 1) (Figs. 1, 2). Follow-up evaluation 6 months after the final therapy was available for 19 of the 34 patients. All seven patients who had PR after 3 months still had PR after 6 months; in 10 of the 12 patients with SD, the evaluation was unchanged, whereas one had a minimal response (MR) and one had PD.

Tumour response (CR or PR) was significantly more frequent in patients whose tumours had a high uptake on the octreoscan [6/7 (86\%) with grade 4 uptake vs. 7/27 $(26 \%)$ with grade 2 or 3 uptake; chi-square test: $P<0.05]$. Five out of the seven patients (71\%) with PD had hepatomegaly and diffuse liver metastases vs 6 out of 27 $(22 \%)$ with CR, PR or SD (chi-square test: $P<0.05)$. Tumour response was more frequent in patients with documented PD within 1 year before the start of the treatment $(8 / 15 ; 53 \%)$ than in those without $(5 / 19 ; 26 \%)$, although this difference was not statistically significant $(P=0.11$; chi-square test). Of the 15 patients with documented PD before the start of the treatment, 3 (20\%) had SD at follow-up. Patients who had a Karnofsky Performance Score of less than 80 before the treatment more frequently had PD $(5 / 8 ; 63 \%)$ than those who had higher scores $(2 / 26 ; 8 \%)(P<0.01$; chi-square test $)$.

The serum tumour marker chromogranin-A was elevated in 29 patients. During the treatment and follow-up, there was a clear decrease in serum chromogranin-A concentrations in patients with PR or CR, whereas these concentrations were virtually unchanged in patients with $\mathrm{SD}$, and initially showed an increase in patients with PD (Fig. 3).

The patient-assessed quality of life, according to the EORTC-QLQ30 questionnaire, was evaluated in 25 patients. Ten patients were excluded because of progressive disease/death $(n=5)$ or missing forms during the followup period ( $n=5$, foreign patients). Scores before the start of the treatment, after receiving $400-600 \mathrm{mCi}{ }^{177} \mathrm{Lu}-$ octreotate and at follow-up 3 months after the final treatment were evaluated. There were no significant differences for functional scales or single symptom scales. The global health scale, on which patients were asked to assign marks regarding both their general health and their quality of life, and which ranged from 0 to 100, was judged as higher than 70 by nine patients $(36 \%)$ before the start of the treatment, by $17(68 \%)$ patients during the treatment and by $16(64 \%)$ patients after the treatment $(P<0.05$; chi-square test $)$. 


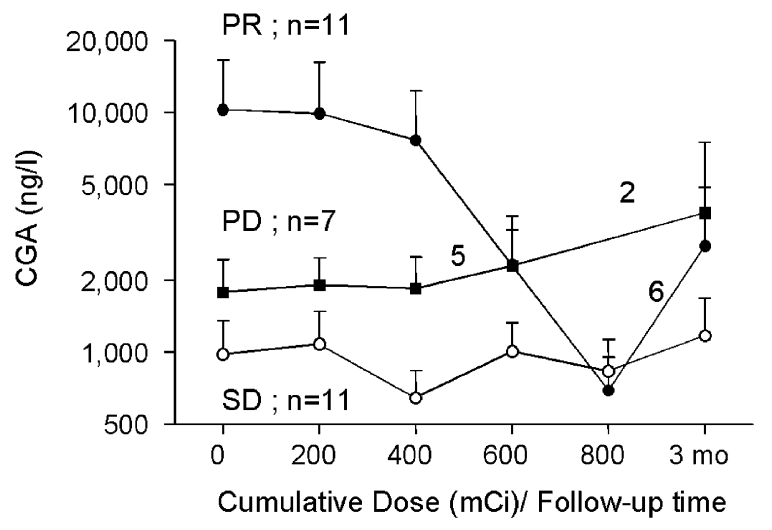

Fig. 3. Serum chromogranin-A concentrations during and after therapy in patients with PR, SD or PD. The reduction in the number of patients during the course of the follow-up was due to death or missing values. Note the logarithmic y-axis

\section{Discussion}

There are few treatment options for metastatic GEP tumours. The use of radiolabelled somatostatin analogues for tumour regression is a promising new development. With ${ }^{90}{ }^{0}$-DOTA ${ }^{0}, \mathrm{Tyr}^{3}$ ] octreotide, PR (or CR) has been reported in 10-25\% of patients with neuroendocrine tumours $[6,7,8]$. In the present study we found objective tumour shrinkage in $38 \%$ of the patients, but PD before the start of the treatment was documented in only $46 \%$ of the patients. This last fact is important, because documented PD was present in more than $80 \%$ of patients in two of the reported series treated with $\left[{ }^{90} \mathrm{Y}-\right.$ DOTA $\left.^{0}, \mathrm{Tyr}^{3}\right]$ octreotide $[6,8]$. As with chemotherapy, an objective tumour response after PRRT is more likely in patients with (fast) growing tumours. Indeed, in our series we also found a trend towards a more favourable treatment outcome in patients with documented PD before the start of the treatment. It may therefore be concluded that the percentage of patients with significant tumour shrinkage in our study might have been even higher had the percentage of patients with PD prior to treatment been comparable to that in studies with $\left[{ }^{90} \mathrm{Y}\right.$ DOTA $^{0}$, Tyr $^{3}$ ]octreotide. The side-effects of treatment with ${ }^{177} \mathrm{Lu}$-octreotate are few and mostly transient, with mild bone marrow depression as the most common finding. Neither renal nor pituitary function deteriorated in any of our patients. Other side-effects can be ascribed to the radiation dose to the testes in men. This dose leads to significantly lower serum testosterone and inhibin-B levels which in turn give rise to higher serum LH and FSH concentrations, thereby substantiating that the pituitary function is unimpaired.

The patient-assessed global health score improved in $30 \%$ of patients during the treatment and the follow-up period. This is an important finding which reflects the improvement in patient well-being and stresses the scarcity of side-effects as perceived by patients. The fact that other scores mainly addressing symptoms did not change significantly is likely due to the diversity of symptoms between patients, the use of Sandostatin by symptomatic patients and the small size of the patient group.

Many physicians adopt an expectant attitude when dealing with patients with metastatic GEP tumours owing to the low success rates of chemotherapy protocols. However, given that treatment with ${ }^{177} \mathrm{Lu}$-octreotate resulted in PR (or CR) in 38\% of our patients, this attitude may be questioned. Although there was a (statistically non-significant) tendency towards more frequent tumour regression in patients who had documented PD before the start of the treatment, those of our patients who had an objective tumour response more frequently had a limited tumour load. This implies that waiting for tumour progression might place patients in a worse position, as PD during or after treatment was more frequent in patients with an extensive tumour load, especially in the liver. We therefore advocate treatment with ${ }^{177} \mathrm{Lu}$-octreotate at an earlier stage of metastatic disease, when even CR may be possible. Another argument in favour of early treatment is that neuroendocrine tumours can dedifferentiate in the course of the disease, and lose their somatostatin receptors, making treatment with radiolabelled somatostatin analogues impossible. If serious side-effects of treatment with ${ }^{177} \mathrm{Lu}$-octreotate remain absent during longer patient follow-up, such treatment could also be considered in an adjuvant setting in patients with neuroendocrine tumours who are operated on with curative intent.

Lastly, as it has been shown in animal experiments that ${ }^{90}$ Y-labelled somatostatin analogues are more effective for larger tumours and ${ }^{177} \mathrm{Lu}$-labelled somatostatin analogues are more effective for smaller tumours [13, 14], combination therapy with ${ }^{90}$ Y-labelled and ${ }^{177} \mathrm{Lu}-\mathrm{la}-$ belled octreotate may be tried in the near future. Such therapy might yield even better results than treatment with either of the radionuclides alone [15].

Acknowledgements. The authors wish to thank all supporting personnel of the Department of Nuclear Medicine for their expert help and effort.

\section{References}

1. Arnold R, Benning R, Neuhaus C, Rolwage M, Trautmann ME. Gastroenteropancreatic endocrine tumours: effect of Sandostatin on tumour growth. The German Sandostatin Study Group. Digestion 1993; 54 (Suppl 1):72-75.

2. Janson ET, Oberg K. Long-term management of the carcinoid syndrome. Treatment with octreotide alone and in combination with alpha-interferon. Acta Oncol 1993; 32:225-229.

3. Ducreux M, Ruszniewski P, Chayvialle JA, Blumberg J, Cloarec D, Michel H, Raymond JM, Dupas JL, Gouerou H, Jian R, Genestin E, Hammel P, Rougier P. The antitumoral effect of the long-acting somatostatin analog lanreotide in neuroendocrine tumors. Am J Gastroenterol 2000; 95:3276-3281. 
4. Valkema R, de Jong M, Bakker WH, Breeman WAP, Kooij PPM, Lugtenburg PJ, de Jong FH, Christiansen A, Kam BLR, de Herder WW, Stridsberg M, Lindemans J, Ensing G, Krenning EP. Phase I study of peptide receptor radionuclide therapy with [111In-DTPA0]octreotide: the Rotterdam experience. Semin Nucl Med 2002; 32:110-122.

5. McCarthy KE, Woltering EA, Espenan GD, Cronin M, Maloney TJ, Anthony LB. In situ radiotherapy with 111Inpentetreotide: initial observations and future directions. Cancer J Sci Am 1998; 4:94-102.

6. Waldherr C, Pless M, Maecke HR, Haldemann A, MuellerBrand J. The clinical value of [90Y-DOTA]-d-Phe1-Tyr3-octreotide (90Y-DOTATOC) in the treatment of neuroendocrine tumours: a clinical phase II study. Ann Oncol 2001; 12:941-945.

7. Paganelli G, Zoboli S, Cremonesi M, Bodei L, Ferrari M, Grana C, Bartolomei M, Orsi F, De Cicco C, Macke HR, Chinol M, de Braud F. Receptor-mediated radiotherapy with 90Y-DOTA-d-Phe1-Tyr3-octreotide. Eur J Nucl Med 2001; 28:426-434.

8. Valkema R, Jamar F, Bakker WH, Norenberg J, Smith C, Stolz B, Kvols L, Pauwels S, Krenning EP. Safety and efficacy of [Y-90-DOTA,Tyr(3)]-octreotide (Y-90-SMT487;OctreoTher) peptide receptor radionuclide therapy (PRRT). Preliminary results of a phase-1 study. Eur J Nucl Med 2001; 28:1025.

9. Reubi JC, Schar JC, Waser B, Wenger S, Heppeler A, Schmitt JS, Macke HR. Affinity profiles for human somatostatin receptor subtypes SST1-SST5 of somatostatin radiotracers selected for scintigraphic and radiotherapeutic use. Eur J Nucl Med 2000; 27:273-282.
10. Erion JL, Bugaj JE, Schmidt MA, Wilhelm RR, Srinivasan A. High radiotherapeutic efficacy of [Lu-177]-DOTA-Y(3)-octreotate in a rat tumor model. J Nucl Med 1999; 40:223P.

11. Kwekkeboom DJ, Bakker WH, Kooij PP, Konijnenberg MW, Srinivasan A, Erion JL, Schmidt MA, Bugaj JL, de Jong M, Krenning EP. [177Lu-DOTA0Tyr3]octreotate: comparison with [111In-DTPA0] octreotide in patients. Eur J Nucl Med 2001; 28:1319-1325.

12. Aaronson NK, Ahmedzai S, Bergman B, Bullinger M, Cull A, Duez A, Filiberti A, Flechtner H, Fleishman SB, de Haes JC. The European Organization for Research and Treatment of Cancer QLQ-C30: a quality-of-life instrument for use in international clinical trials in oncology. J Natl Cancer Inst 1993; 85:365-376

13. De Jong M, Breeman WA, Bernard BF, Bakker WH, Schaar M, van Gameren A, Bugaj JE, Erion J, Schmidt M, Srinivasan A, Krenning EP. [177Lu-DOTA(0),Tyr3]octreotate for somatostatin receptor-targeted radionuclide therapy. Int J Cancer 2001; 92:628-633.

14. De Jong M, Breeman WA, Bernard BF, Bakker WH, Visser TJ, Kooij PP, van Gameren A, Krenning EP. Tumor response after [(90)Y-DOTA(0),Tyr(3)]octreotide radionuclide therapy in a transplantable rat tumor model is dependent on tumor size. J Nucl Med 2001; 42:1841-1846.

15. De Jong M, Valkema R, Jamar F, Kvols LK, Kwekkeboom DJ, Breeman WAP, Bakker WH, Smith C, Pauwels S, Krenning EP. Somatostatin receptor-targeted radionuclide therapy of tumors: preclinical and clinical findings. Semin Nucl Med 2002; 32:133-140. 